\title{
THE FRENET VECTOR FIELDS AND THE CURVATURES OF THE NATURAL LIFT CURVE
}

\author{
Evren ERGÜN ${ }^{1}$, Mustafa BíLiCí ${ }^{2}$ and Mustafa ÇALIŞKAN ${ }^{3}$ \\ ${ }^{1}$ Ondokuz Mayıs University Faculty of Arts and Sciences Department of \\ Mathematics,Samsun, Turkey. \\ ${ }^{2}$ Ondokuz Mayıs University Educational Faculty Department of Mathematics,Samsun, \\ Turkey \\ ${ }^{3}$ Gazi University Faculty of Sciences Department of Mathematics, Ankara, Turkey. \\ ${ }^{1}$ eergun@omu.edu.tr, ${ }^{2}$ mbilici@omu.edu.tr, ${ }^{3}$ mustafacaliskan@gazi.edu.tr
}

Keywords: Natural Lift, Frenet Frame, Curvature, Torsion

Abstract: In this paper, Frenet vector fields, curvature and torsion of the natural lift curve of a given curve is calculated by using the angle between Darboux vector field and the binormal vector field of the given curve in $\square_{1}^{3}$. Also, a similar calculation is made in $\square_{1}^{3}$ considering timelike or spacelike Darboux vector field.

\section{Introduction}

Thorpe gave the concepts of the natural lift curve and geodesic spray in [7]. Thorpe provied the natural lift $\bar{\alpha}$ of the curve $\alpha$ is an integral curve of the geodesic spray iff $\alpha$ is an geodesic on M in [7].Çalışkan, Sivridağ and Hacısalihoğlu studied the natural lift curves of the spherical indicatries of tangent, principal normal, binormal vectors and fixed centrode of a curve in [2]. They gave some interesting results about the original curve were obtaied, depending on the assumption that the natural lift curve should be the integral curve of the geodesic spray on the tangent bundle $\mathrm{T}\left(\mathrm{S}^{2}\right)$ in [2]. Ergün and Çalışkan defined the concepts of the natural lift curve and geodesic spray in Minkowski 3-space in [6]. The anologue of the theorem of Thorpe was given in Minkowski 3-space by Ergün and Çalışkan in [3]. Walrave characterized the curve with constant curvature in Minkowski 3-space in [8].

Let $\alpha: I \subset \square \rightarrow \square^{3}$ be regular curve with arclength parameter s. $\langle$,$\rangle is the standard scalar$ product of $\square^{3}$ given by

$$
\langle\mathrm{X}, \mathrm{Y}\rangle=\mathrm{x}_{1} \mathrm{y}_{1}+\mathrm{x}_{2} \mathrm{y}_{2}+\mathrm{x}_{3} \mathrm{y}_{3}
$$

for each vectors $\mathrm{X}=\left(\mathrm{x}_{1}, \mathrm{x}_{2}, \mathrm{x}_{3}\right), \mathrm{Y}\left(\mathrm{y}_{1}, \mathrm{y}_{2}, \mathrm{y}_{3}\right) \in \square^{3} \because$ The norm of a vector $\mathrm{X}=\left(\mathrm{x}_{1}, \mathrm{x}_{2}, \mathrm{x}_{3}\right) \in \square^{3}$ is defined by

$$
\|\mathrm{X}\|=\sqrt{\langle\mathrm{X}, \mathrm{X}\rangle},[1]
$$

Let $\alpha: \mathrm{I} \subset \square \rightarrow \square^{3}$ be a parametrized curve. We denote by $\{\mathrm{T}(\mathrm{s}), \mathrm{N}(\mathrm{s}), \mathrm{B}(\mathrm{s})\}$ the moving Frenet frame along the curve $\alpha$, where $\mathrm{T}, \mathrm{N}$ and $\mathrm{B}$ are the tangent, the principal normal and the binormal vector of the curve $\alpha$, respectively.

Let $\alpha$ be a reguler curve in $\square^{3}$. Then

$$
\mathrm{T}=\frac{\dot{\alpha}}{\|\dot{\alpha}\|}, \mathrm{N}=\mathrm{B} \times \mathrm{T}, \mathrm{B}=\frac{\dot{\alpha} \times \ddot{\alpha}}{\|\dot{\alpha} \times \ddot{\alpha}\|},[1] .
$$

Let $\alpha$ be a unit speed space curve with curvature $\mathrm{K}$ and torsion $\tau$, then

$$
\kappa=\frac{\|\dot{\alpha} \times \ddot{\alpha}\|}{\|\dot{\alpha}\|^{3}} \text { and } \tau=\frac{\operatorname{det}(\dot{\alpha}, \ddot{\alpha}, \ddot{\alpha})}{\|\dot{\alpha} \times \ddot{\alpha}\|^{2}} \text {. }
$$


If $\alpha$ is a unit speed curve,then

$$
\mathrm{T}=\dot{\alpha}, \mathrm{N}=\frac{\ddot{\alpha}}{\|\ddot{\alpha}\|}, \mathrm{B}=\mathrm{T} \times \mathrm{N},[1] .
$$

Let $\alpha$ be a unit speed space curve with curvature $\mathrm{K}$ and torsion $\tau$ and let Frenet vector fields of $\alpha$ be $\{\mathrm{T}, \mathrm{N}, \mathrm{B}\}$. We can write

$$
\mathrm{T} \times \mathrm{N}=\mathrm{B}, \mathrm{N} \times \mathrm{B}=\mathrm{T}, \mathrm{B} \times \mathrm{T}=\mathrm{N},
$$

where $\times$ the Euclidean cross product in space $\square^{3}$. Then, Frenet formulas are given by

$$
\dot{\mathrm{T}}=\kappa \mathrm{N}, \dot{\mathrm{N}}=-\kappa \mathrm{T}+\tau \mathrm{B}, \dot{\mathrm{B}}=-\tau \mathrm{N},[1] \text {. }
$$

For any unit speed curev $\alpha: \mathrm{I} \subset \square \rightarrow \square^{3}$, we call $\mathrm{W}(\mathrm{s})=\tau \mathrm{T}(\mathrm{s})+\kappa \mathrm{B}(\mathrm{s})$ the Darboux vector field of $\alpha$. $\theta$ being an angle between $B$ and the Frenet instantaneous rotation vector $W$, we can write

$$
\kappa=\|\mathrm{W}\| \cos \theta, \tau=\|\mathrm{W}\| \sin \theta
$$

Let $\mathrm{M}$ be a hypersurface in $\square^{3}$ and let $\alpha: \mathrm{I} \rightarrow \mathrm{M}$ be a parametrized curve M. $\alpha$ is called an integral curve of $\mathrm{X}$ if

$$
\frac{\mathrm{d}}{\mathrm{ds}}(\alpha(\mathrm{s}))=\mathrm{X}(\alpha(\mathrm{s})),(\text { for all } \mathrm{s} \in \mathrm{I}),[4]
$$

where $X$ is a smooth tangent vector field on $M$. We have $T M=\bigcup_{P \in M} T_{P} M=\chi(M)$, where $T_{P} M$ is the tangent space of $\mathrm{M}$ at $\mathrm{P}$ and $\mathrm{M} \chi \mathrm{M}$ is the space of vector fields on $\mathrm{M}$.

For any parametrized curve $\alpha: \mathrm{I} \rightarrow \mathrm{M}, \bar{\alpha}: \mathrm{I} \rightarrow \mathrm{TM}$ given by

$$
\bar{\alpha}(\mathrm{s})=(\alpha(\mathrm{s}), \dot{\alpha}(\mathrm{s}))=\left.\dot{\alpha}(\mathrm{s})\right|_{\alpha(\mathrm{s})}
$$

is called the natural lift of $\alpha$ on TM. Thus, we can write

$$
\frac{\mathrm{d} \bar{\alpha}}{\mathrm{d} s}=\frac{\mathrm{d}}{\mathrm{ds}}\left(\left.\dot{\alpha}(\mathrm{s})\right|_{\alpha(s)}\right)=\mathrm{D}_{\dot{\alpha}(\mathrm{s})} \dot{\alpha}(\mathrm{s})
$$

where D is the Levi-Civita connection on $\square^{3},[7]$.

Let Minkowski 3-space $\square_{1}^{3}$ be the vector space $\square^{3}$ equipped with the Lorentzian inner product g given by

where $\mathrm{X}=\left(\mathrm{x}_{1}, \mathrm{x}_{2}, \mathrm{x}_{3}\right) \in \square^{3}$. A vector $\mathrm{X}=\left(\mathrm{x}_{1}, \mathrm{x}_{2}, \mathrm{x}_{3}\right) \in \square_{1}^{3}$ is said to be timelike if $\mathrm{g}(\mathrm{X}, \mathrm{X})<0$, spacelike if $g(X, X)>0$ and lightlike (or null) if $(X, X)=0$. Similarly, an arbitrary curve $\alpha=\alpha(\mathrm{t})$ in ${ }_{1}^{3}$ where $\mathrm{t}$ is a pseudo-arclength parameter, can locally be timelike, spacelike or null (lightlike), if all of its velocity vectors $\alpha(\mathrm{t})$ are respectively timelike, spacelike or null (lightlike), for every $t \in I \subset \square$. A lightlike vector $X$ is said to be positive (resp. negative) if and only if $x_{1}>0$ (resp. $\mathrm{x}_{1}<0$ ) and a timelike vector $\mathrm{X}$ is said to be positive (resp. negative) if and only if $\mathrm{x}_{1}>0$ (resp. $\mathrm{x}_{1}<0$ ). The norm of a vector $\mathrm{X}$ is defined by

$$
\|\mathrm{X}\|_{\mathrm{IL}}=\sqrt{|\mathrm{g}(\mathrm{X}, \mathrm{X})|},[5]
$$

Let $\alpha: \mathrm{I} \rightarrow \square_{1}^{3}$ be a parametrized curve. We denote by $\{\mathrm{T}(\mathrm{s}), \mathrm{N}(\mathrm{s}), \mathrm{B}(\mathrm{s})\}$ the moving Frenet frame along the curve $\alpha$, where T,N and B are the tangent, the principal normal and the binormal vector of the curve $\alpha$, respectively.

Let $\alpha$ be a unit speed timelike space curve with curvature $\mathrm{K}$ and torsion $\tau$. Let Frenet vector fields of $\alpha$ be $\{\mathrm{T}, \mathrm{N}, \mathrm{B}\}$. In this trihedron, $\mathrm{T}$ is timelike vector field, $\mathrm{N}$ and $\mathrm{B}$ are spacelike vector fields.For this vectors, we can write

$$
\mathrm{T} \times \mathrm{N}=\mathrm{B}, \mathrm{N} \times \mathrm{B}=-\mathrm{T}, \mathrm{B} \times \mathrm{T}=\mathrm{N}
$$

where $\times$ is the Lorentzian cross product in space $\square_{1}^{3}$. Then, Frenet formulas are given by 


$$
\dot{\mathrm{T}}=\kappa \mathrm{N}, \dot{\mathrm{N}}=\kappa \mathrm{T}+\tau \mathrm{B}, \dot{\mathrm{B}}=-\tau \mathrm{N},[8]
$$

Let $\alpha$ be a unit speed spacelike space curve with a spacelike binormal. In this trihedron, we assume that $\mathrm{T}$ and $\mathrm{B}$ are spacelike vector fields and $\mathrm{N}$ is a timelike vector field In this situation,.

$$
\mathrm{T} \times \mathrm{N}=\mathrm{B}, \mathrm{N} \times \mathrm{B}=\mathrm{T}, \mathrm{B} \times \mathrm{T}=-\mathrm{N},
$$

Then, Frenet formulas are given by

$$
\dot{\mathrm{T}}=\kappa \mathrm{N}, \dot{\mathrm{N}}=\kappa \mathrm{T}+\tau \mathrm{B}, \dot{\mathrm{B}}=\tau \mathrm{N},[8] .
$$

Let $\alpha$ be a unit speed spacelike space curve with a spacelike binormal. In this trihedron, we assume that $\mathrm{T}$ and $\mathrm{N}$ are spacelike vector fields and $\mathrm{B}$ is a timelike vector field.In this situation,.

$$
\mathrm{T} \times \mathrm{N}=-\mathrm{B}, \mathrm{N} \times \mathrm{B}=\mathrm{T}, \mathrm{B} \times \mathrm{T}=\mathrm{N},
$$

Then, Frenet formulas are given by,

$$
\dot{\mathrm{T}}=\kappa \mathrm{N}, \dot{\mathrm{N}}=-\kappa \mathrm{T}+\tau \mathrm{B}, \dot{\mathrm{B}}=\tau \mathrm{N},[8] \text {. }
$$

Lemma 1: Let $X$ and $Y$ be nonzero Lorentz orthogonal vectors in $\square_{1}^{3}$. If $X$ is timelike, then $Y$ is spacelike,[6].

Lemma 2: Let $X$ and $Y$ be pozitive (negative ) timelike vectors in $\square_{1}^{3}$. Then

$$
\mathrm{g}(\mathrm{X}, \mathrm{Y}) \leq\|\mathrm{X}\|\|\mathrm{Y}\|
$$

whit equality if and only if $\mathrm{X}$ and $\mathrm{Y}$ are linearly dependent.

\section{Lemma 3:}

(i) Let $\mathrm{X}$ and $\mathrm{Y}$ be pozitive (negative ) timelike vectors in $\square_{1}^{3}$. By the Lemma 2, there is unique nonnegative real number $\varphi(X, Y)$ such that

$$
\mathrm{g}(\mathrm{X}, \mathrm{Y}) \leq\|\mathrm{X}\|\|\mathrm{Y}\| \cosh \varphi(\mathrm{X}, \mathrm{Y})
$$

the Lorentzian timelike angle between $\mathrm{X}$ and $\mathrm{Y}$ is defined to be $\varphi(\mathrm{X}, \mathrm{Y})$.

ii) Let $X$ and $Y$ be spacelike vektors in $\square_{1}^{3}$ that span a spacelike vector subspace. Then we have

$$
|\mathrm{g}(\mathrm{X}, \mathrm{Y})| \leq\|\mathrm{X}\|\|\mathrm{Y}\| \text {. }
$$

Hence, there is a unique real number $\varphi(X, Y)$ between 0 and $\pi$ such that

$$
\mathrm{g}(\mathrm{X}, \mathrm{Y})=\|\mathrm{X}\|\|\mathrm{Y}\| \cos \varphi(\mathrm{X}, \mathrm{Y})
$$

the Lorentzian spacelike angle between $\mathrm{X}$ and $\mathrm{Y}$ is defined to be $\varphi(\mathrm{X}, \mathrm{Y})$.

(iii) Let $X$ and $Y$ be spacelike vectors in $\square_{1}^{3}$ that span a timelike vector subspace. Then we have

$$
\mathrm{g}(\mathrm{X}, \mathrm{Y})>\|\mathrm{X}\|\|\mathrm{Y}\| \text {. }
$$

Hence, there is a unique pozitive real number $\varphi(X, Y)$ between 0 and $\pi$ such that

$$
|\mathrm{g}(\mathrm{X}, \mathrm{Y})|=\|\mathrm{X}\|\|\mathrm{Y}\| \cosh \varphi(\mathrm{X}, \mathrm{Y})
$$

the Lorentzian timelike angle between $\mathrm{X}$ and $\mathrm{Y}$ is defined to be $\varphi(\mathrm{X}, \mathrm{Y})$.

(iv) Let $X$ be a spacelike vector and $Y$ be a pozitive timelike vector in $\mathbb{R}_{1}{ }^{3}$. Then there is a unique nonnegative reel number $\varphi(X, Y)$ such that

$$
|\mathrm{g}(\mathrm{X}, \mathrm{Y})|=\|\mathrm{X}\|\|\mathrm{Y}\| \sinh \varphi(\mathrm{X}, \mathrm{Y})
$$

the Lorentzian timelike angle between $\mathrm{X}$ and $\mathrm{Y}$ is defined to be $\varphi(\mathrm{X}, \mathrm{Y}),[6]$.

Definition1. (Unit Vector $C$ of Direction $W$ for Non-null Curves):

i)For the curve $\alpha$ with a timelike tanget, $\theta$ being a Lorentzian timelike angle between the spacelike binormal unit - $\mathrm{B}$ and the Frenet instantaneous rotation vector $\mathrm{W}$,

a) If $|\kappa|>|\tau|$, then $W$ is a spacelike vector. In this situation, from Lemma 3 iii) we can write

$$
\kappa=\|\mathrm{W}\| \cosh \theta, \tau=\|\mathrm{W}\| \sinh \theta
$$

$\|\mathrm{W}\|^{2}=\mathrm{g}(\mathrm{W}, \mathrm{W})=\kappa^{2}-\tau^{2}$ and $\mathrm{C}=\frac{\mathrm{W}}{\|\mathrm{W}\|}=\sinh \theta \mathrm{T}+\cosh \theta \mathrm{B}$, where $\mathrm{C}$ is unit vector of direction W. 
b) If $|\kappa|<|\tau|$, then $\mathrm{W}$ is a timelike vector. In this situation, from Lemma 3 iv) we can write

$$
\kappa=\|\mathrm{W}\| \sinh \theta, \tau=\|\mathrm{W}\| \cosh \theta
$$

$\|\mathrm{W}\|^{2}=-\mathrm{g}(\mathrm{W}, \mathrm{W})=-\left(\kappa^{2}-\tau^{2}\right)$ and $\mathrm{C}=\cosh \theta \mathrm{T}+\sinh \theta \mathrm{B}$.

ii) For the curve $\alpha$ with a timelike principal normal, $\theta$ being an angle between the $B$ and the $W$, if $B$ and $\mathrm{W}$ spacelike vectors that span a spacelike vector subspace then by the Lemma 3

ii) we can write

$$
\kappa=\|\mathrm{W}\| \cos \theta, \tau=\|\mathrm{W}\| \sin \theta
$$

$\|\mathrm{W}\|^{2}=\mathrm{g}(\mathrm{W}, \mathrm{W})=\kappa^{2}+\tau^{2}$ and $\mathrm{C}=\sin \theta \mathrm{T}-\cos \theta \mathrm{B}$.

iii) For the curve $\alpha$ with a timelike binormal, $\theta$ being a Lorentzian timelike angle between the -B and the $\mathrm{W}$,

a) If $|\kappa|<|\tau|$, then $W$ is a spacelike vector. In this situation, from Lemma 3 iv) we can write

$$
\kappa=\|\mathrm{W}\| \sinh \theta, \tau=\|\mathrm{W}\| \cosh \theta
$$

$\|\mathrm{W}\|^{2}=-\mathrm{g}(\mathrm{W}, \mathrm{W})=-\left(\tau^{2}-\kappa^{2}\right)$ and $\mathrm{C}=-\sinh \theta \mathrm{T}+\cosh \theta \mathrm{B}$.

Let $\mathrm{M}$ be a hypersurface in $\square_{1}^{3}$ and let $\alpha: \mathrm{I} \rightarrow \mathrm{M}$ be a parametrized curve. $\alpha$ is called an integral curve of $\mathrm{X}$ if

$$
\frac{\mathrm{d}}{\mathrm{ds}}(\alpha(\mathrm{s}))=\mathrm{X}(\alpha(\mathrm{s})),(\text { for all } \mathrm{s} \in \mathrm{I})
$$

where $X$ is a smooth tangent vector field on $M$, [5]. We have $T M=\bigcup_{P \in M} T_{P} M=\chi(M)$ where $T_{P} M$ is the tangent space of $\mathrm{M}$ at $\mathrm{P}$ and $\chi(\mathrm{M})$ is the space of vector fields on $\mathrm{M}$.

For any parametrized curve $\alpha: \mathrm{I} \rightarrow \mathrm{M}, \bar{\alpha}: \mathrm{I} \rightarrow \mathrm{TM}$ given by

$$
\bar{\alpha}(s)=(\alpha(s), \dot{\alpha}(s))=\left.\dot{\alpha}(s)\right|_{\alpha(s)}
$$

is called the natural lift of $\alpha$ on TM. Thus, we can write

$$
\frac{\mathrm{d} \bar{\alpha}}{\mathrm{ds}}=\frac{\mathrm{d}}{\mathrm{ds}}\left(\left.\dot{\alpha}(\mathrm{s})\right|_{\alpha(s)}\right)=\mathrm{D}_{\alpha(\mathrm{s})} \dot{\alpha}(\mathrm{s})
$$

where $\mathrm{D}$ is the Levi-Civita connection on $\square_{1}^{3},[3]$.

For any parametrized curve in $\square^{3} \alpha: I \rightarrow M, \bar{\alpha}: I \rightarrow T M$ given by

$$
\bar{\alpha}(\mathrm{s})=(\alpha(\mathrm{s}), \dot{\alpha}(\mathrm{s}))=\left.\dot{\alpha}(\mathrm{s})\right|_{\alpha(s)}
$$

is called the natural lift of $\alpha$ on TM, [7].

We denote by $\{\overline{\mathrm{T}}(\mathrm{s}), \overline{\mathrm{N}}(\mathrm{s}), \overline{\mathrm{B}}(\mathrm{s})\}$ the moving Frenet frame along the curve $\bar{\alpha}$, where $\bar{T}, \bar{N}$ and $\bar{B}$ are the tangent, the principal normal and the binormal vector of the curve $\bar{\alpha}$, respectively.

Corollary1: Let $\bar{\alpha}$ be the natural lift of $\square$ in $\square^{3}$ and be a reguler curve. Then

$$
\begin{aligned}
& \overline{\mathrm{T}}(\mathrm{s})=\mathrm{N}(\mathrm{s}) \\
& \overline{\mathrm{N}}(\mathrm{s})=-\cos \theta \mathrm{T}(\mathrm{s})+\sin \theta \mathrm{B}(\mathrm{s}) \\
& \overline{\mathrm{B}}(\mathrm{s})=\sin \theta \mathrm{T}(\mathrm{s})+\cos \theta \mathrm{B}(\mathrm{s}) .
\end{aligned}
$$

Corollary2: Let $\alpha$ be the natural lift of $\alpha$ with curvature $\overline{\mathrm{K}}$ and torsion $\bar{\tau}$. Then

$$
\bar{\kappa}=\frac{1}{\cos \theta}, \bar{\tau}=\frac{\dot{\theta}}{\|\mathrm{W}\| \cos \theta} .
$$

For any parametrized curve in $\square_{1}^{3} \alpha: \mathrm{I} \rightarrow \mathrm{M}, \bar{\alpha}: \mathrm{I} \rightarrow \mathrm{TM}$ given by

$$
\bar{\alpha}(\mathrm{s})=(\alpha(\mathrm{s}), \dot{\alpha}(\mathrm{s}))=\left.\dot{\alpha}(\mathrm{s})\right|_{\alpha(s)}
$$


is called the natural lift of $\alpha$ on TM, [3].

We denote by $\{\overline{\mathrm{T}}(\mathrm{s}), \overline{\mathrm{N}}(\mathrm{s}), \overline{\mathrm{B}}(\mathrm{s})\}$ the moving Frenet frame along the curve $\bar{\alpha}$, where $\bar{T}, \bar{N}$ and $\bar{B}$ are the tangent, the principal normal and the binormal vector of the curve $\bar{\alpha}$, respectively.

Corollary3: Let $\alpha$ be a unit speed timelike space curve and $\bar{\alpha}$ be the natural lift of $\alpha$.If $\mathrm{W}$ is a spacelike vector field, then

$$
\begin{aligned}
& \overline{\mathrm{T}}(\mathrm{s})=\mathrm{N}(\mathrm{s}) \\
& \overline{\mathrm{N}}(\mathrm{s})=-\cosh \theta \mathrm{T}(\mathrm{s})-\sinh \theta \mathrm{B}(\mathrm{s}) \\
& \overline{\mathrm{B}}(\mathrm{s})=-\sinh \theta \mathrm{T}(\mathrm{s})-\cosh \theta \mathrm{B}(\mathrm{s}) .
\end{aligned}
$$

Corollary4: Let $\alpha$ be a unit speed timelike space curve and the natural lift $\bar{\alpha}$ of the curve $\alpha$ be a space curve with with curvature $\bar{\kappa}$ and torsion $\bar{\tau}$. If W is a spacelike vector field, then

$$
\bar{\kappa}=\frac{1}{\cosh \theta}, \bar{\tau}=-\frac{\dot{\theta}}{\|\mathrm{W}\| \cosh \theta} .
$$

Corollary5: Let $\alpha$ be a unit speed timelike space curve and $\bar{\alpha}$ be the natural lift of $\alpha$.If $\mathrm{W}$ is a timelike vector field, then

$$
\begin{aligned}
& \overline{\mathrm{T}}(\mathrm{s})=\mathrm{N}(\mathrm{s}) \\
& \overline{\mathrm{N}}(\mathrm{s})=-\sinh \theta \mathrm{T}(\mathrm{s})-\cosh \theta \mathrm{B}(\mathrm{s}) \\
& \overline{\mathrm{B}}(\mathrm{s})=-\cosh \theta \mathrm{T}(\mathrm{s})-\sinh \theta \mathrm{B}(\mathrm{s}) .
\end{aligned}
$$

Corollary6: Let $\alpha$ be a unit speed timelike space curve and the natural lift $\bar{\alpha}$ of the curve $\alpha$ be a space curve with curvature $\bar{\kappa}$ and torsion $\bar{\tau}$. If W is a timelike vector field, then

$$
\bar{\kappa}=\frac{1}{\sinh \theta}, \bar{\tau}=\frac{\dot{\theta}}{\|\mathrm{W}\| \sinh \theta} .
$$

Corollary7: Let $\alpha$ be a unit speed spacelike space curve with a spacelike binormal and $\bar{\alpha}$ be the natural lift of $\alpha$.Then

$$
\begin{aligned}
& \overline{\mathrm{T}}(\mathrm{s})=\mathrm{N}(\mathrm{s}) \\
& \overline{\mathrm{N}}(\mathrm{s})=\cos \theta \mathrm{T}(\mathrm{s})+\sin \theta \mathrm{B}(\mathrm{s}) \\
& \overline{\mathrm{B}}(\mathrm{s})=\sin \theta \mathrm{T}(\mathrm{s})-\cos \theta \mathrm{B}(\mathrm{s}) .
\end{aligned}
$$

Corollary8: Let $\alpha$ be a unit speed spacelike space curve with a spacelike binormal and the natural lift $\bar{\alpha}$ of the curve $\alpha$ be a space curve with curvature $\bar{\kappa}$ and torsion $\bar{\tau}$. Then

$$
\bar{\kappa}=\frac{1}{\cos \theta}, \bar{\tau}=-\frac{\dot{\theta}}{\|\mathrm{W}\| \cos \theta} .
$$

Corollary9: Let $\alpha$ be a unit speed spacelike space curve with a timelike binormal and $\bar{\alpha}$ be the natural lift of $\alpha$. If $\mathrm{W}$ is a spacelike vector field, then

$$
\begin{aligned}
& \overline{\mathrm{T}}(\mathrm{s})=\mathrm{N}(\mathrm{s}) \\
& \overline{\mathrm{N}}(\mathrm{s})=\sinh \theta \mathrm{T}(\mathrm{s})-\cosh \theta \mathrm{B}(\mathrm{s}) \\
& \overline{\mathrm{B}}(\mathrm{s})=\cosh \theta \mathrm{T}(\mathrm{s})-\sinh \theta \mathrm{B}(\mathrm{s}) .
\end{aligned}
$$

Corollary10: Let $\alpha$ be a unit speed spacelike space curve with a timelike binormal and the natural lift $\bar{\alpha}$ of the curve $\alpha$ be a space curve with curvature $\bar{\kappa}$ and torsion $\bar{\tau}$. If W is a spacelike vector field, then

$$
\bar{\kappa}=\frac{1}{\sinh \theta}, \bar{\tau}=-\frac{\dot{\theta}}{\|\mathrm{W}\| \sinh \theta} .
$$


Corollary11: Let $\alpha$ be a unit speed spacelike space curve with a timelike binormal and $\bar{\alpha}$ be the natural lift of $\alpha$. If $\mathrm{W}$ is a timelike vector field, then

$$
\begin{aligned}
& \overline{\mathrm{T}}(\mathrm{s})=\mathrm{N}(\mathrm{s}) \\
& \overline{\mathrm{N}}(\mathrm{s})=\cosh \theta \mathrm{T}(\mathrm{s})-\sinh \theta \mathrm{B}(\mathrm{s}) \\
& \overline{\mathrm{B}}(\mathrm{s})=\sinh \theta \mathrm{T}(\mathrm{s})-\cosh \theta \mathrm{B}(\mathrm{s}) .
\end{aligned}
$$

Corollary12: Let $\alpha$ be a unit speed spacelike space curve with a timelike binormal and the natural lift $\bar{\alpha}$ of the curve $\alpha$ be a space curve with curvature $\bar{\kappa}$ and torsion $\bar{\tau}$. If $\mathrm{W}$ is a timelike vector field, then

$$
\bar{\kappa}=\frac{1}{\cosh \theta}, \bar{\tau}=\frac{\dot{\theta}}{\|\mathrm{W}\| \cosh \theta} .
$$

\section{References}

[1] Carmo M. P. Do, 1976, Differential Geometry of Curve Surfaces.Prentice-Hall, Inc., Englewood Cliffs, New Jersey

[2] Çalışkan M. Sivridağ A.İ. and Hacısalihoğlu H.H., (1984) Some Characterizationsfor the natural lift curves and the geodesic spray, Communications, Fac. Sci.Univ. Ankara Ser. A Math. 33, Num. 28,235-242.

[3] Ergün E., Çalışkan M., (2011), On Geodesic Sprays In Minkowski 3-Space,Int. Journal of Contemp. Math. Sciences, Vol. 6, no. 39, 1929-1933.

[4] O'Neill B., 1967, Elementery Differential Geometry Academic Press, New York and London.

[5] O'Neill B., 1983, Semi-Riemannian Geometry, with applications to relativity.Academic Press, New York.

[6] Ratcliffe J.G., 1994, Foundations of Hyperbolic Manifolds, Springer-Verlag, New York, Inc., New York.

[7] Thorpe J.A., 1979, Elementary Topics In Differential Geometry,Springer-Verlag, New York, Heidelberg-Berlin.

[8] Walrave J., 1995, Curves and Surfaces in Minkowski Space K. U. Leuven Faculteit Der Wetenschappen. 